\title{
Belgeo
}

Revue belge de géographie

\section{Étude des impacts hydrauliques, sédimentologiques et écologiques liés aux travaux hydrauliques sur la Semois}

Hydraulic, sedimentological and ecological impacts of the riverbed dredgings in the Semois catchment (Belgian Ardenne)

F. Gob, G. Houbrechts, J. Mols, F. Petit, F. Guyon, F. Rosillon, X. Cogels, P. Vander Borght, J.P. Ancion, E. Ntibarufata, J.M. Hiver et J.C. Philippart

\section{OpenEdition \\ Journals}

Édition électronique

URL : https://journals.openedition.org/belgeo/16584

DOI : $10.4000 /$ belgeo. 16584

ISSN : 2294-9135

Éditeur :

National Committee of Geography of Belgium, Société Royale Belge de Géographie

Édition imprimée

Date de publication : 30 septembre 2003

Pagination : 243-256

ISSN : 1377-2368

Référence électronique

F. Gob, G. Houbrechts, J. Mols, F. Petit, F. Guyon, F. Rosillon, X. Cogels, P. Vander Borght, J.P. Ancion, E. Ntibarufata, J.M. Hiver et J.C. Philippart, «Étude des impacts hydrauliques, sédimentologiques et écologiques liés aux travaux hydrauliques sur la Semois », Belgeo [En ligne], 3 | 2003, mis en ligne le 30 octobre 2003, consulté le 21 septembre 2021. URL : http://journals.openedition.org/belgeo/16584 ; DOI : https://doi.org/10.4000/belgeo.16584

Ce document a été généré automatiquement le 21 septembre 2021.

Belgeo est mis à disposition selon les termes de la licence Creative Commons Attribution 4.0 International. 


\title{
Étude des impacts hydrauliques, sédimentologiques et écologiques liés aux travaux hydrauliques sur la Semois
}

\author{
Hydraulic, sedimentological and ecological impacts of the riverbed dredgings in \\ the Semois catchment (Belgian Ardenne)
}

F. Gob, G. Houbrechts, J. Mols, F. Petit, F. Guyon, F. Rosillon, X. Cogels, P. Vander Borght, J.P. Ancion, E. Ntibarufata, J.M. Hiver et J.C. Philippart

1 Confrontée à des inondations fréquentes, la Semois a fait l'objet de différentes interventions visant, principalement, à entretenir les ouvrages d'art, à lutter contre l'érosion et à réduire les inondations. Ces travaux d'aménagement ont des incidences, tant sur la morphologie du lit du cours d'eau que sur la faune et la flore de cet écosystème. Ce dernier est constitué, schématiquement, du lit mineur comprenant la bande active constituée de bancs alluviaux peu ou pas végétalisés, et le lit majeur ou plaine inondable. Ces écosystèmes, généralement interconnectés et dont l'origine, la structure et l'évolution sont intimement liées à la dynamique fluviale actuelle ou passée, peuvent être interprétés comme les éléments d'un système hydrofluvial plus complexe.

2 Les approches mises en œuvre pour étudier les influences des travaux d'aménagement ne peuvent donc se limiter à l'utilisation des outils classiques de l'hydraulique. Elles doivent intégrer l'ensemble des caractéristiques hydro-environnementales du système. Afin de cerner l'importance de ces impacts et fournir un outil d'aide à la décision pour les gestionnaires du cours d'eau, une méthodologie d'évaluation interdisciplinaire a été mise en place. Un tronçon expérimental de la Semois ardennaise, long de près de $40 \mathrm{~km}$ (situé entre le village de Dohan et celui de Laviot) a été sélectionné pour sa représentativité (Fig. 1). Le travail a été réalisé à l'échelle de ce tronçon, mais également à des échelles plus locales, par sélection de sites ateliers. Le site de Laviot en est un exemple: il s'est révélé particulièrement riche en enseignement grâce à un 
important dragage réalisé en 1997 sur un linéaire de près de $1 \mathrm{~km}$. Plusieurs études ont été menées sous la direction du Laboratoire de Recherches hydrauliques du MET. Il s'agit d'une étude des impacts sédimentologiques et géomorphologiques (Laboratoire d'Hydrogra phie et de Géomorphologie fluviatile, ULg), d'une étude des impacts hydrauliques (Laboratoire de Recherches hydrauliques) et d'une étude des impacts hydrobiologiques et écologiques (FUL et Laboratoire de Démographie des poissons et d'hydroécologie ULg).

Figure 1. Localisation du site d'étude, La Semois entre Dohan et Laviot.

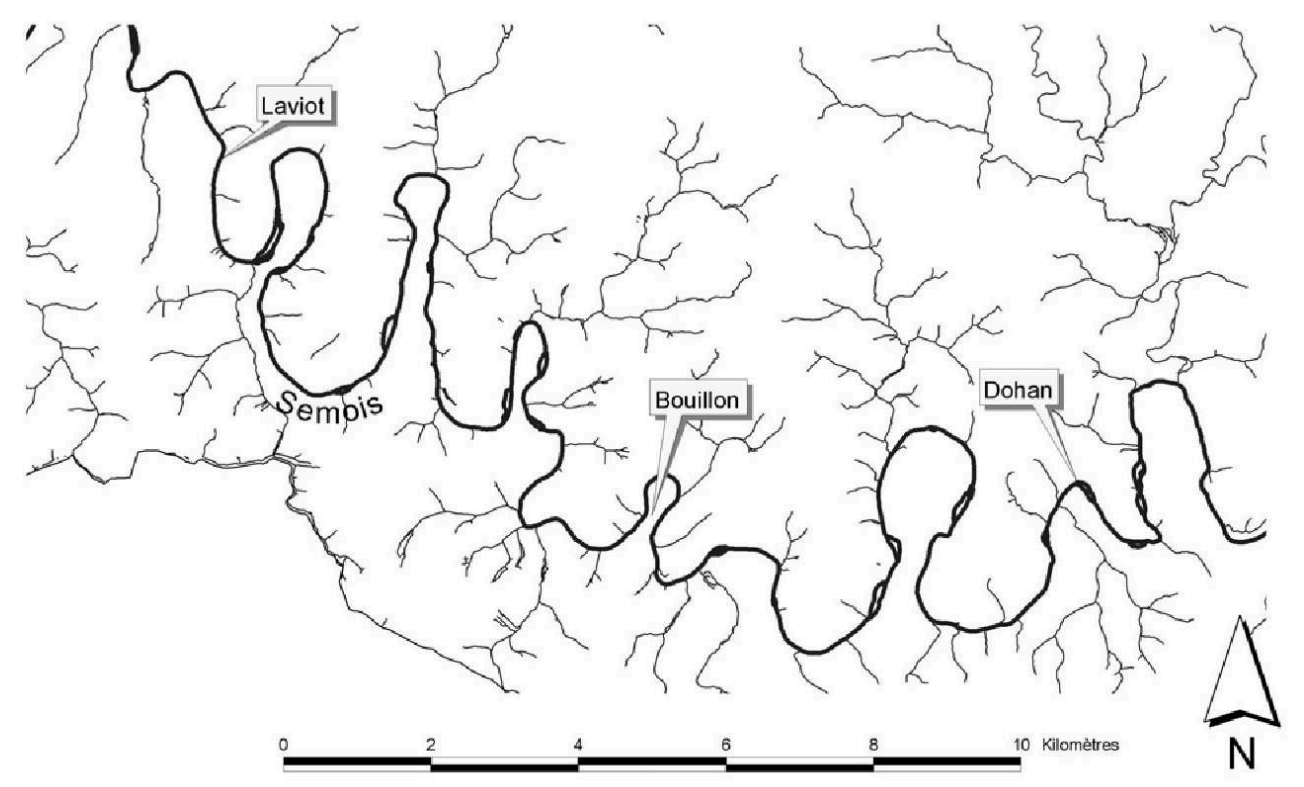

\section{Aspects sédimentologiques et géomorphologiques}

Les grands méandres encaissés de la Semois ardennaise induisent un fonctionnement $\mathrm{du}$ système fluvial nettement différent de celui de rivières mieux connues du nord du Massif ardennais. En outre, étant donné la forme même du bassin hydrographique de la Semois, ses affluents, spécialement dans la partie ardennaise de son cours, sont de dimension modeste mais présentent de forts gradients. La Semois se distingue également par le confinement de sa plaine alluviale qui empêche le développement de méandres libres, par le nombre très limité de larges dépôts émergés en étiage et par la présence de nombreux affleurements de roche en place dans le lit mineur. Ceci influence la morphologie du lit mineur. Ainsi, par rapport aux rivières du nord de l'Ardenne telles que l'Ourthe ou la Lesse, la profondeur moyenne de la Semois au plein bord est moindre, son rapport largeur profondeur plus élevé ( 30$)$ et son débit à plein bord est atteint plus rapidement : il est de l'ordre de $130 \mathrm{~m}^{3} \mathrm{~s}-1$ à la station de Membre et a une récurrence de 1,1 an. Par contre, le débit à plein bord des principaux affluents situés dans le secteur d'étude est atteint plus rarement que sur la Semois même, ce qui va à l'encontre du schéma généralement observé dans des bassins hydrographiques homogènes et hiérarchisés (Petit et al., 1996).

Dans la gestion des sédiments et notamment pour cerner l'impact des travaux de dragages et leur efficacité, il était nécessaire de déterminer le taux de renouvellement 
des formes, c'est à dire d'estimer le temps nécessaire pour qu'il y ait reconstitution des dépôts et pour que le lit retrouve son aspect initial. Pour ce faire, il convenait de mettre en évidence le débit mobilisateur de la charge de fond, d'estimer la taille du matériau mobilisé et d'identifier les sources de sédiments (érosion latérale, incision dans la nappe alluviale, apport des affluents).

Une première approche consistait à suivre la dynamique de la rivière grâce à une analyse diachronique réalisée selon une méthodologie classique (cartes topographiques anciennes, cartes postales, photographies aériennes...). Cette étude a montré que l'évolution du lit mineur en deux siècles est peu importante : absence de méandration active au sein de la plaine alluviale, érosion latérale limitée, rareté des bancs émergés, que ce soit en position de convexité ou aux points d'inflexion. La seule évolution sensible, s'observe au niveau de la dynamique des îles qui évoluent le plus souvent par colmatage de l'un des bras. De plus, ces îles sont actuellement végétalisées ce qui trahit un ralentissement de leur activité et est plus que probablement l'indice d'un héritage.

6 Afin de déterminer le débit de mise en mouvement de la charge de fond, la taille des matériaux mobilisés et la distance moyenne parcourue, différentes campagnes de marquage ont été réalisées sur la Semois et sur les affluents. Plusieurs techniques ont été utilisées: (i) marquage colorimétrique in situ (ce qui évite la déstabilisation des matériaux) et par injection (ce qui permet un taux de récupération plus élevé); (ii) injections d'éléments métalliques (galets plombés), technique qui permet de retrouver un grand nombre d'éléments, même enfouis; (iii) marquage au moyen d'émetteurs radio (les galets marqués peuvent être suivis pendant la crue).

7 Les résultats issus des différentes techniques citées ci-dessus indiquent que le débit de mobilisation de la charge de fond dans la Semois est proche du débit à plein bord, ce qui s'oppose à nouveau aux observations effectuées sur des rivières du nord de l'Ardenne où la mobilisation s'observe déjà pour des crues nettement inférieures au plein bord $(0.5 \mathrm{Qb})$ (Gob et al., 2003). Par ailleurs, il y a simultanéité entre les crues mobilisatrices de la Semois et celles des affluents d'une part et adéquation entre la charge de fond mobilisée dans les affluents et dans la Semois d'autre part $(\mathrm{D} 50=60 \mathrm{~mm})$. De ce fait, la charge injectée par les affluents est évacuée par la Semois, sans décalage sensible au niveau d'une saison hydrologique.

8 Les forces tractrices et les puissances spécifiques de la Semois (au débit à plein bord et lors de crues de forte récurrence) sont nettement moindres que dans les rivières du nord de l'Ardenne (Gob et al., 2003). Mais, dans la Semois, la rugosité globale est moins importante que sur les autres rivières de dimension comparable (le coefficient de rugosité totale de Manning atteint à peine 0,045 contre 0,060 sur l'Ourthe inférieure par exemple). Ainsi, dans la décomposition des forces tractrices totales, la part de la bedform shear stress est moindre dans la Semois, laissant plus de disponibilité à la grain shear stress, la seule qui intervient dans la mobilisation et le transport des sédiments. Cette moindre rugosité de la Semois s'explique par des alternances des formes du lit moins développées, du fait de l'absence de méandres libres et de la limitation des profondeurs liée aux nombreux affleurements de roche en place. En outre, la largeur plus importante du lit mineur de la Semois (cf. rapport w/d) assure probablement une plus large bande active de charriage.

9 Le débit solide de la charge de fond de la Semois a pu être estimé grâce à une série de relevés topographiques du talweg réalisés à Laviot, où un important dragage a été effectué en septembre 1997. Un chenal central avec approfondissement local de près de 
$2 \mathrm{~m}$ sur une largeur de $20 \mathrm{~m}$ a été creusé, plus de $11000 \mathrm{~m}^{3}$ de sédiments ont été retirés. Quatre séries de 18 profils transversaux ont été effectués systématiquement aux mêmes endroits : en 1997 juste avant les travaux, en 1997 juste après les travaux, en 2001 et en 2002 ; ils ont permis de suivre le comblement partiel de la fosse créée par le curage (fig. 2 et 3$)$.

Figure 2. Profils en long de la Semois à Laviot effectués en 1997 (avant curage), en 1997 (après curage), 2001.

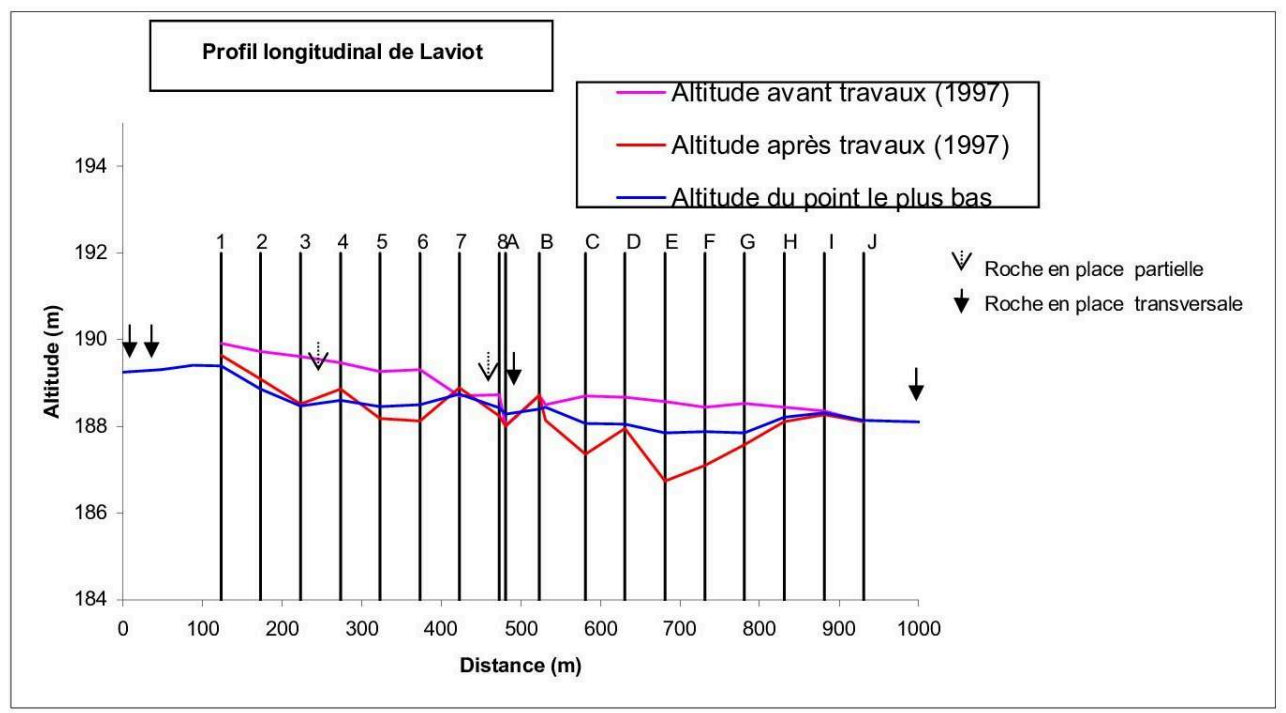

Figure 3. Profils en travers de la Semois à Laviot effectués en 1997 (avant curage), en 1997 (après curage), 2001 et 2002 . Une fosse de plus de $2 \mathrm{~m}$ de profondeur a été creusée et presque entièrement comblée 4 ans après.

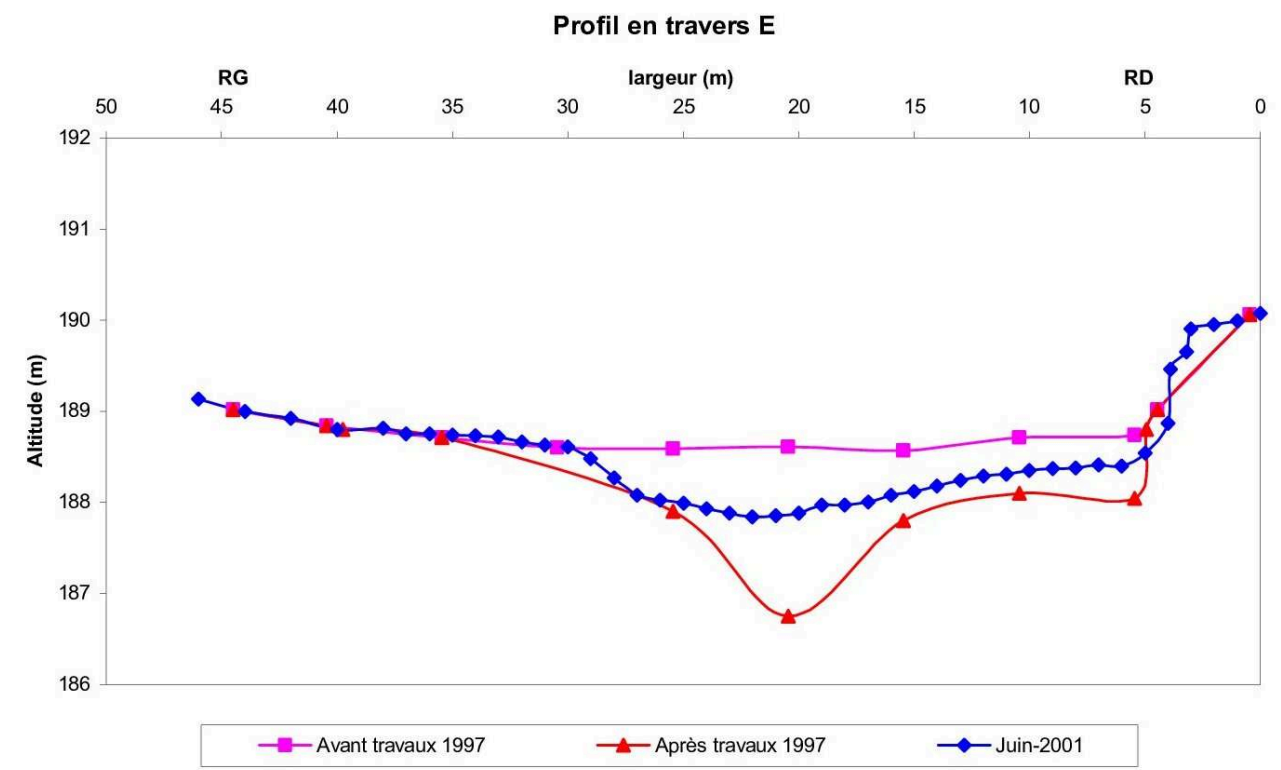

10 Étant donné son ampleur, cette fosse a joué le rôle de piège à sédiments : entre 1997 et 2000 , le stock sédimentaire ne s'est reconstitué qu'à raison de $5500 \mathrm{~m}^{3}$, ceci malgré l'occurrence de plusieurs crues mobilisatrices. L'importance du flux sédimentaire nous permet d'estimer un retour à l'état initial (avant dragage) après environ 10 ans. Malgré 
l'importance énorme du dragage réalisé en 1997, ceci ne représente qu'une solution temporaire (et limitée) aux problèmes d'inondation.

11 A partir de ces données et d'autres dragages effectués systématiquement dans les mêmes sites, nous avons pu estimer le débit solide de la charge de fond de la Semois à 1,1 t.km-2.an-1. A nouveau, la Semois se démarque des rivières de taille comparable du nord de l'Ardenne qui ont des valeurs de débit solide deux fois moins importantes (entre 0,4 et 0,5 t.km-2.an-1 ), (Petit et al., 1996), ceci malgré des forces tractrices et des puissances spécifiques plus faibles mais une rugosité moins importante.

\section{L'aspect hydraulique}

Pour mener à bien son étude des impacts hydrauliques, le Laboratoire de Recherches hydrauliques a développé un modèle numérique unidimensionnel d'écoulement permanent (Bertrand, 1994). Dans ce modèle, la contribution du lit majeur est prise en compte grâce au calcul de pertes de charge par débitance. L'interaction entre le lit mineur et le lit majeur est étudiée par un ajustement de la débitance des sections. Les données géométriques proviennent de levés topographiques qui étaient tout d'abord disponibles. Les débits utilisés pour les simulations sont ceux mesurés par le Service d'Etudes hydrologiques lors de précédentes crues. Différentes mesures de niveau d'eau proviennent des laisses de crue et des témoignages des riverains.

Le modèle a été appliqué, en tenant compte des données existantes, mais il ne donnait pas entière satisfaction. Après une première approximation, il s'avérait qu'il devait être affiné. A cette fin, des levés supplémentaires ont été réalisés dans le tronçon expérimental, et un réseau de limnimètres et de limnigraphes a été installé. Les crues suivantes, surtout celle très importante de janvier 2001, ont permis de calibrer le modèle pour en faire un outil fiable qui pourra aider les décideurs à prendre des mesures efficaces (fig. 4).

Figure 4. Modélisation de la crue du 16 janvier 2001 grâce à l'axe hydraulique développé par le Laboratoire de recherche hydraulique du M.E.T.

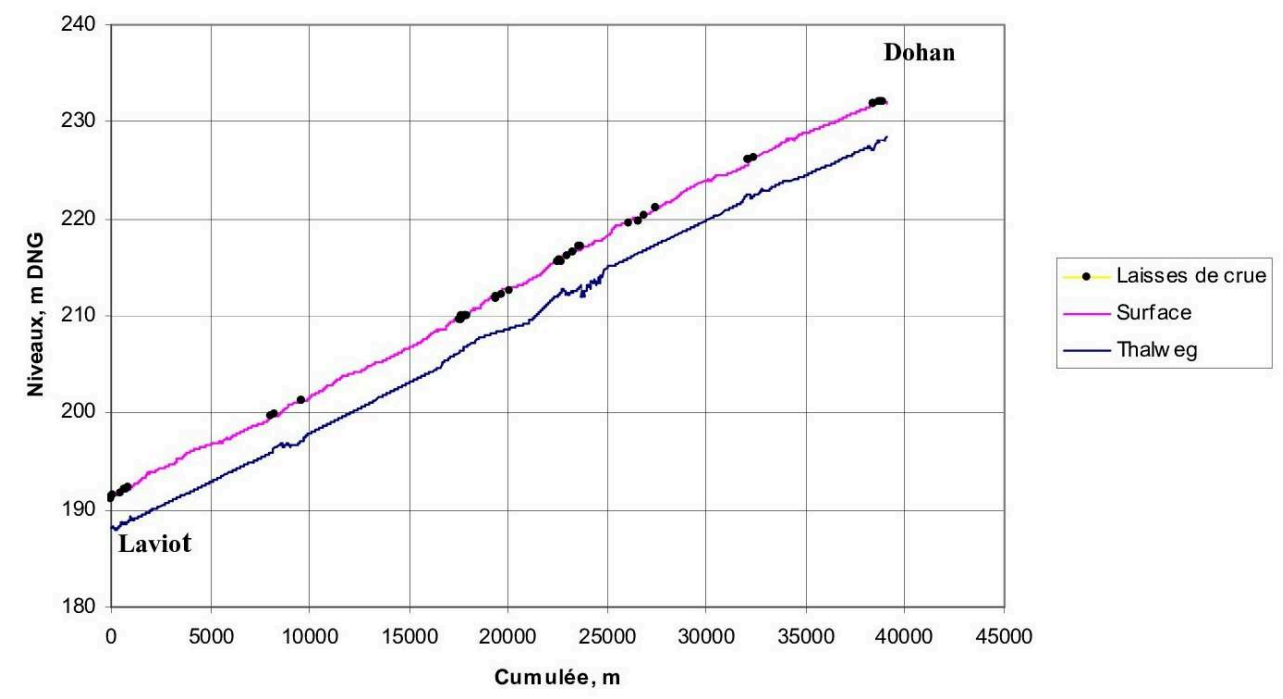



Bouillon (juste en amont du barrage à aiguilles); la commune avait émis l'idée qu'un curage à cet endroit pourrait diminuer l'effet des inondations. L'étude hydraulique a prouvé que les effets étaient tout à fait négligeables, quelques centimètres tout au plus, et uniquement à l'endroit du curage.

Le second cas, particulièrement exemplaire, concerne Laviot. En 1997, un curage important constituant en un retrait de plus de 11000 mètres cubes sur une longueur de 800 mètres avait eu lieu à cet endroit. Les travaux avaient été arrêtés suite à l'intervention de plusieurs associations défendant l'environnement. Après la crue importante de janvier 2001, les riverains s'étaient plaints du fait que l'inondation à cet endroit aurait pu être évitée si les travaux de curage réalisés quatre ans plus tôt s'étaient prolongés vers l'aval.

16 Les études hydrauliques réalisées en utilisant notre "outil » ont démontré que le curage à cet endroit ne provoque qu'une diminution de niveau d'eau de quelques centimètres en cas de crue et qu'une prolongation des curages vers l'aval aurait eu des effets négligeables.

17 De même, une digue construite en cet endroit pour protéger les riverains de la crue précitée devrait atteindre 1,70 mètres de haut, ce qui est impensable dans une zone touristique comme la Semois (fig. 5).

18 En général, on remarque que les curages locaux dans la Semois ont peu d'effet pour la protection des inondations. Cela est notamment dû à la rugosité élevée. Pour avoir un effet de diminution de niveau importante, il faudrait curer la rivière sur plusieurs kilomètres, ce qui est évidemment impossible, vu l'impact sur l'environnement.

Figure 5. Simulation des axes hydrauliques à Laviot en considérant une digue pour la protection contre les inondations.

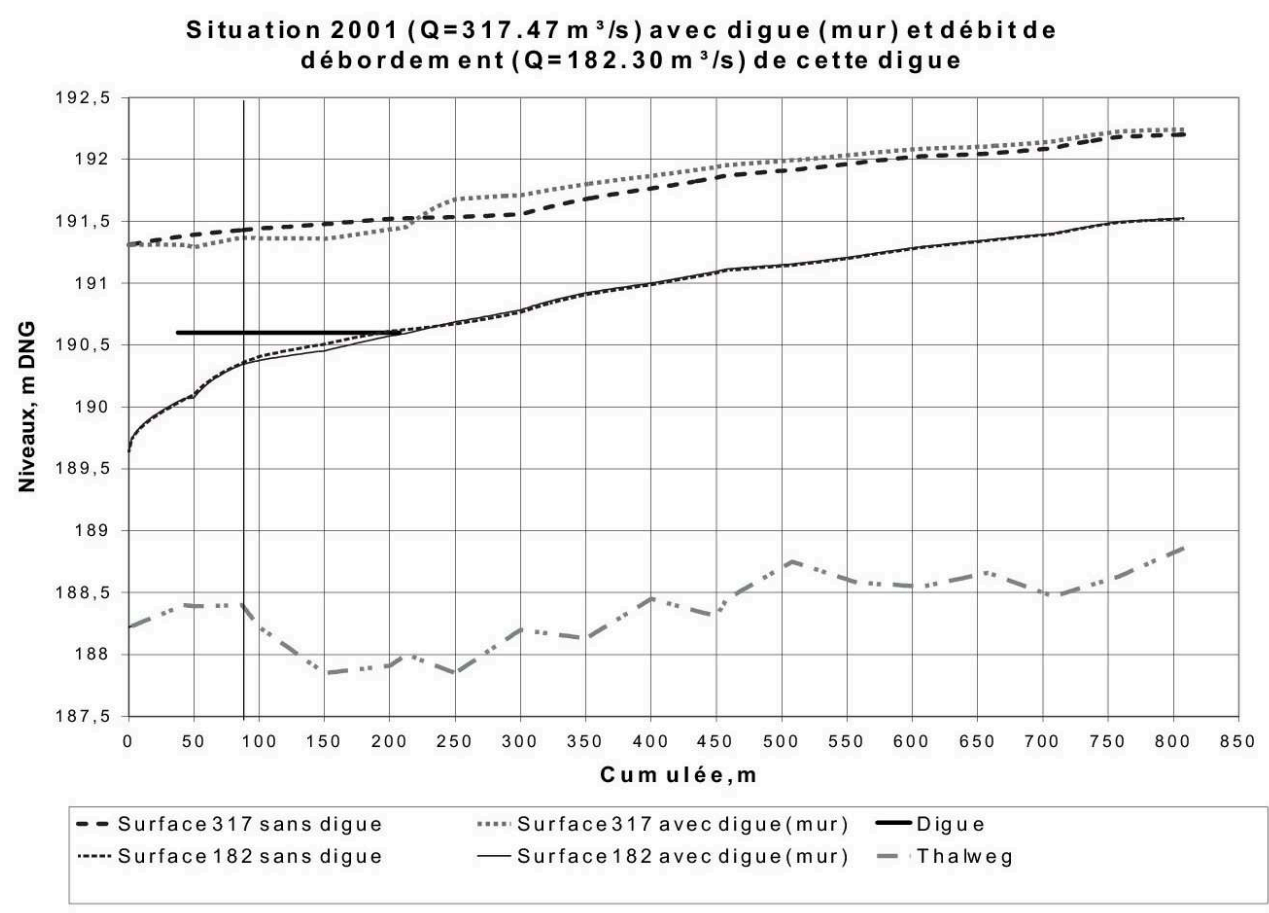

Belgeo, 3 | 2003 


\section{L'aspect environnemental}

\section{Un objectif, des échelles d'analyse et des outils}

19 Cette étude confiée à la FUL avait pour objectif la validation d'outils d'estimation des impacts écologiques liés aux travaux en basse Semois. Deux échelles d'approches de la problématique ont été abordées : l'échelle du tronçon et l'échelle stationnelle ou locale.

Différents outils ont été testés à ces deux échelles afin :

- d'établir un état de référence de la qualité écologique de la rivière en termes de qualité physicochimique des eaux (outil Seq.Eau développé par les Agences de l'Eau en France), de qualité biologique (IBGN, indice diatomique réalisés par L. Leclercq de l'ULg), de qualité physique globale du cours d'eau et de sa plaine alluviale (outil QUALPHY développé par l'Agence de l'Eau Rhin-Meuse), de description des milieux et des espèces présents (données biologiques existantes) et de caractérisation du contexte environnemental (contraintes naturelles et réglementaires, pressions et usages).

- de valider des méthodologies d'estimation des impacts de travaux dans le lit du cours d'eau sur l'habitat piscicole (outil EVHA développé par le CEMAGREF de Lyon selon Ginot et Souchon, 1998).

\section{Approche de l'habitat piscicole à l'échelle locale}

21 Celle-ci a été amorcée dans le cadre de cette étude en termes d'estimation de la perte d'habitabilité du cours d'eau suite à des travaux hydrauliques (homogénéisation des faciès d'écoulement, perte de la diversité de ces faciès...) qui constitue un impact majeur sur les peuplements piscicoles. Elle est pertinente car elle est en lien direct avec les deux autres approches hydraulique et sédimentologique envisagées dans le cadre de cette étude.

22 A l'échelle locale (cas de Laviot), nous avons testé en collaboration avec le Laboratoire de Démographie des Poissons et d'Hydroécologie, la méthodologie des microhabitats et le logiciel EVHA développés par le CEMAGREF de Lyon (Ginot et al., 1998). Cet organisme s'est intéressé depuis quelques années aux différentes méthodes d'approche de l'habitat physique des poissons et notamment à la méthode «IFIM » ou Instream Flow Incremental Methodology développée par l'US Fish \& Wildlife Service, Fort Collins (Colorado) au début des années 1980 (Stalnaker, 1979; Bovee, 1982). Cette méthode permet d'estimer et d'analyser un potentiel d'habitat sur la base de quelques variables morphodynamiques (hauteur, vitesse et substrat), et ce de façon dynamique en incluant une dimension temporelle, la variation des débits. Elle couple ainsi un modèle hydraulique caractérisant ces variables avec un modèle biologique définissant les préférences d'utilisation de ces variables morphodynamiques pour des espèces cibles indicatrices selon leur stade de développement et éventuellement selon leurs fonctions (nutrition, repos, reproduction).

23 L'espèce indicatrice choisie est le barbeau fluviatile. En effet, cette espèce est une des deux espèces les plus représentatives de l'ichtyomasse de la Basse Semois (avec le chevaine). Cette espèce est de plus typique de la zone à barbeau caractéristique de la rivière selon la classification de Huet (1949). Phillippart et al. (1994) précisent dans leur étude que «le barbeau est représentatif des espèces lithophiles (pondeurs sur substrat pierreux; classification de Balon, 1975), guilde reproductrice à laquelle appartient de 
nombreuses espèces comme le chevaine, le hotu, le vairon, l'ablette spirlin, l'ombre commun... » (espèces présentes en Basse Semois). Ils signalent également que "des études précédentes (dont Baras, 1992) ont mis en évidence des recouvrements importants entre les aires de frayère $d u$ barbeau et de l'ombre, du chevaine ou du vairon". Ces divers arguments justifient le choix $\mathrm{du}$ barbeau fluviatile comme «espèce modèle indicatrice ». Le modèle biologique utilisé dans le test de l'outil EVHA sur la Semois provient donc d'études sur l'Ourthe réalisées par l'ULg (contexte très proche de celui de la Semois navigable). Il correspond aux courbes d'utilisation des trois variables d'habitat retenues par la méthode par l'espèce indicatrice choisie, en l'occurrence le barbeau fluviatile. Les graphiques des figures 6 et 7 présentent les courbes d'utilisation des variables «hauteur d'eau» et "vitesse du courant " chez le barbeau fluviatile adulte (> à $15 \mathrm{~cm}$ ) dans l'Ourthe, en fonction de trois activités de l'espèce, d'après Phillippart et al. (1994).

Figures 6 et 7 . Courbes d'utilisation des variables « hauteur d'eau » et « vitesse du courant » chez le barbeau adulte dans l'Ourthe, en fonction de trois activités de l'espèce.

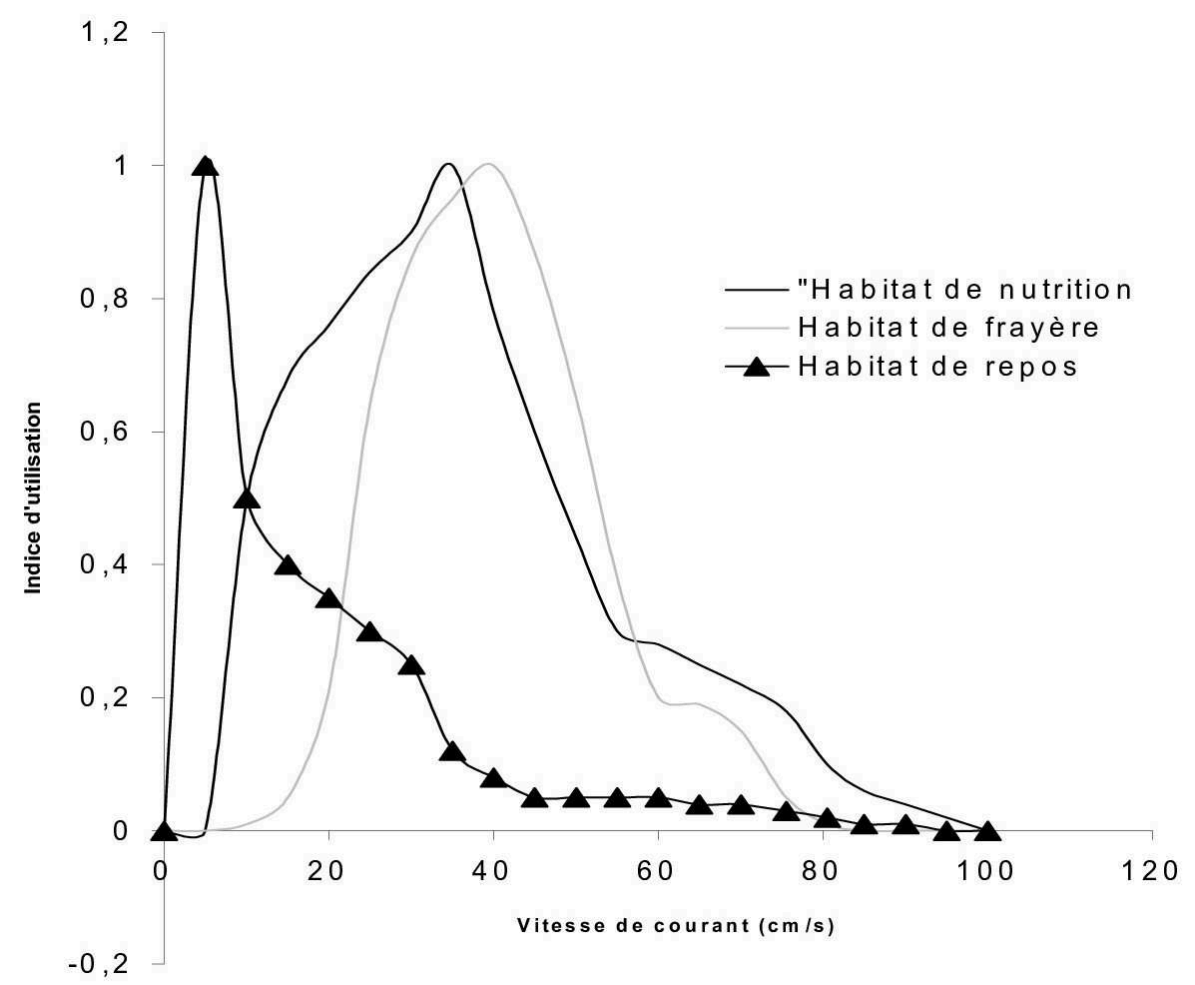




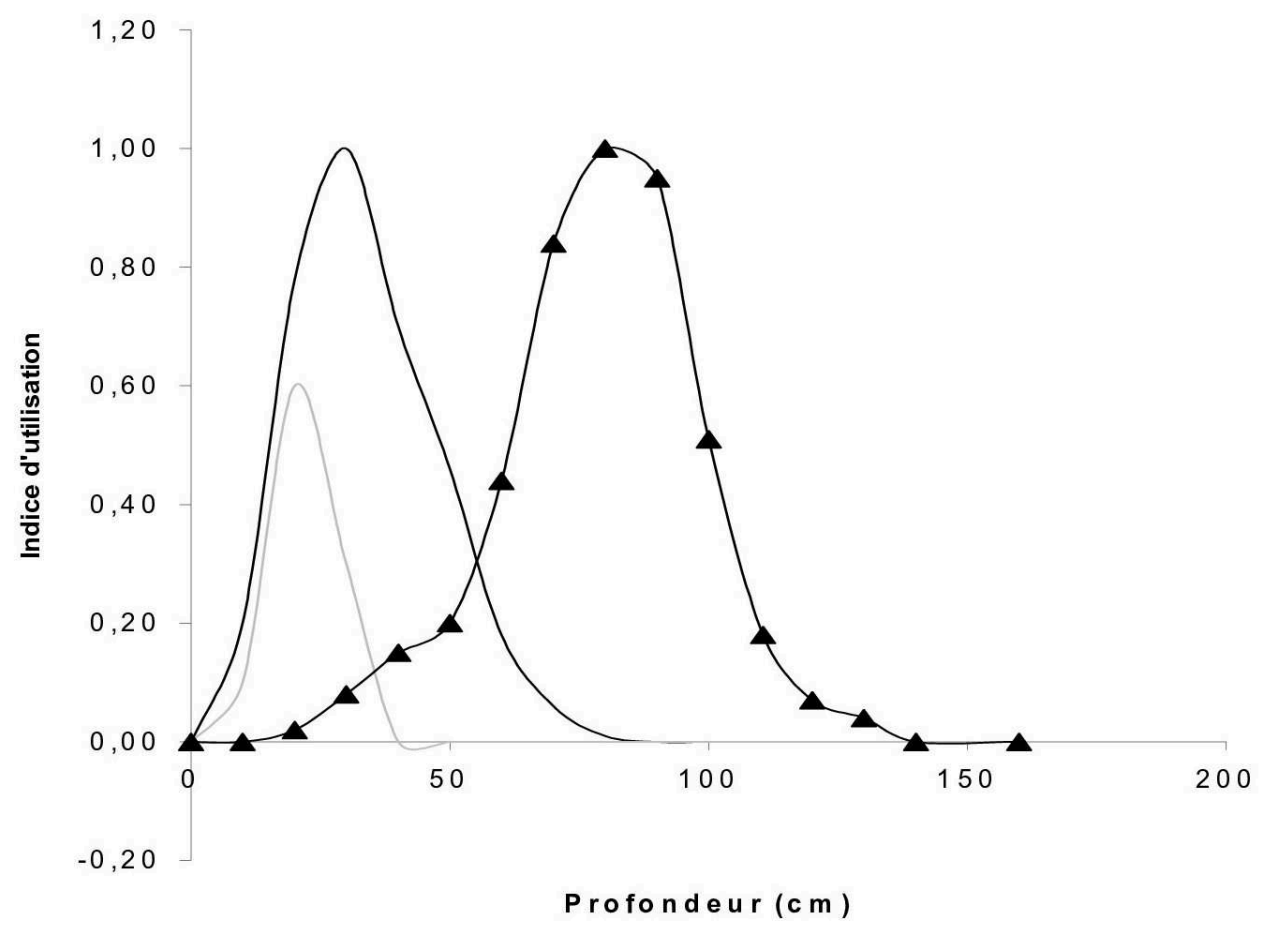

D’après Phillippart et al. (1994) mouillée.

Ces deux graphiques mettent notamment en évidence que l'habitat de nutrition du barbeau adulte est composé préférentiellement de zones présentant une profondeur et une vitesse de courant moyennes (faciès de plat). Par contre, son habitat de repos implique plutôt des faciès de cours d'eau plus profonds et des vitesses faibles (faciès de mouilles ou de chenal lent).

La collecte de données sur le terrain implique :

- la reconnaissance des unités morphodynamiques (faciès) présents au sein de la station à l'étude sur base de la typologie établie par Malavoi (1989);

- la prise de mesures topographiques et hydrauliques (hauteur, vitesses et substrat) le long de transects (en moyenne 3 transects perpendiculaires à l'écoulement par faciès). Ces données permettent de caler le modèle hydraulique.

Sur base de ces données, le logiciel reconstruit la géométrie de la rivière et partitionne l'aire d'étude en cellules homogènes caractérisées par des valeurs spécifiques des 3 variables morphodynamiques. Le couplage avec le modèle biologique permet le calcul de la Surface Potentiellement Utilisable (SPU) pour l'espèce visée au stade de croissance considéré et pour une fonction donnée (nutrition, repos, reproduction), caractérisant ainsi la capacité d'accueil du milieu pour cette espèce. La valeur d'habitat, également calculée par le logiciel, correspond au rapport entre la valeur de SPU et la surface

Les résultats obtenus lors de l'application de la méthode au site de Laviot sont présentés aux figures 8 et 9 . 
Figures 8 et 9 . Estimation de la Surface Potentielle Utilisable par le Barbeau fluviatile adulte en termes d'habitat de nutrition pour les 6 transects de mesures de la station Laviot3 en Semois navigable.

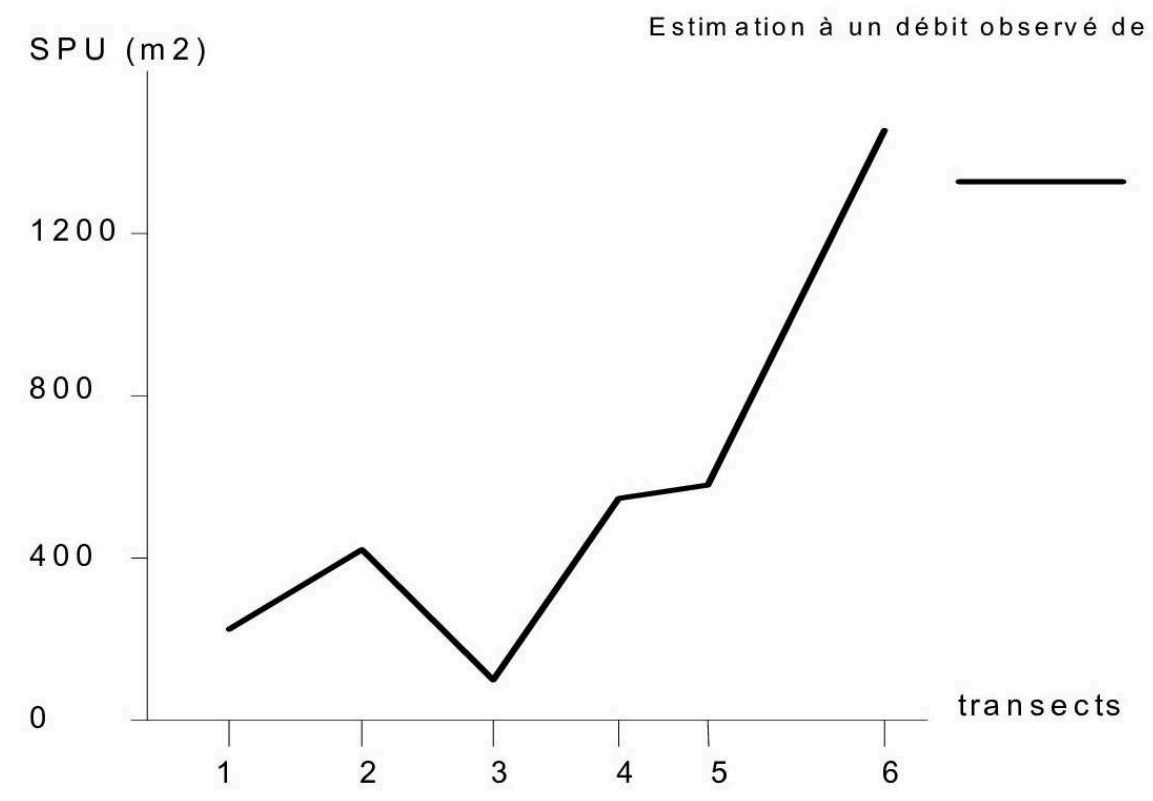

Valeurs d'habitat globales et partielles calculées par le logiciel EVHA pour la station Laviot 3 (Semois navigable)

Espèce indicatrice : Barbeau fluviatile adulte Type d'habitat pris en compte : habitat de nutrition
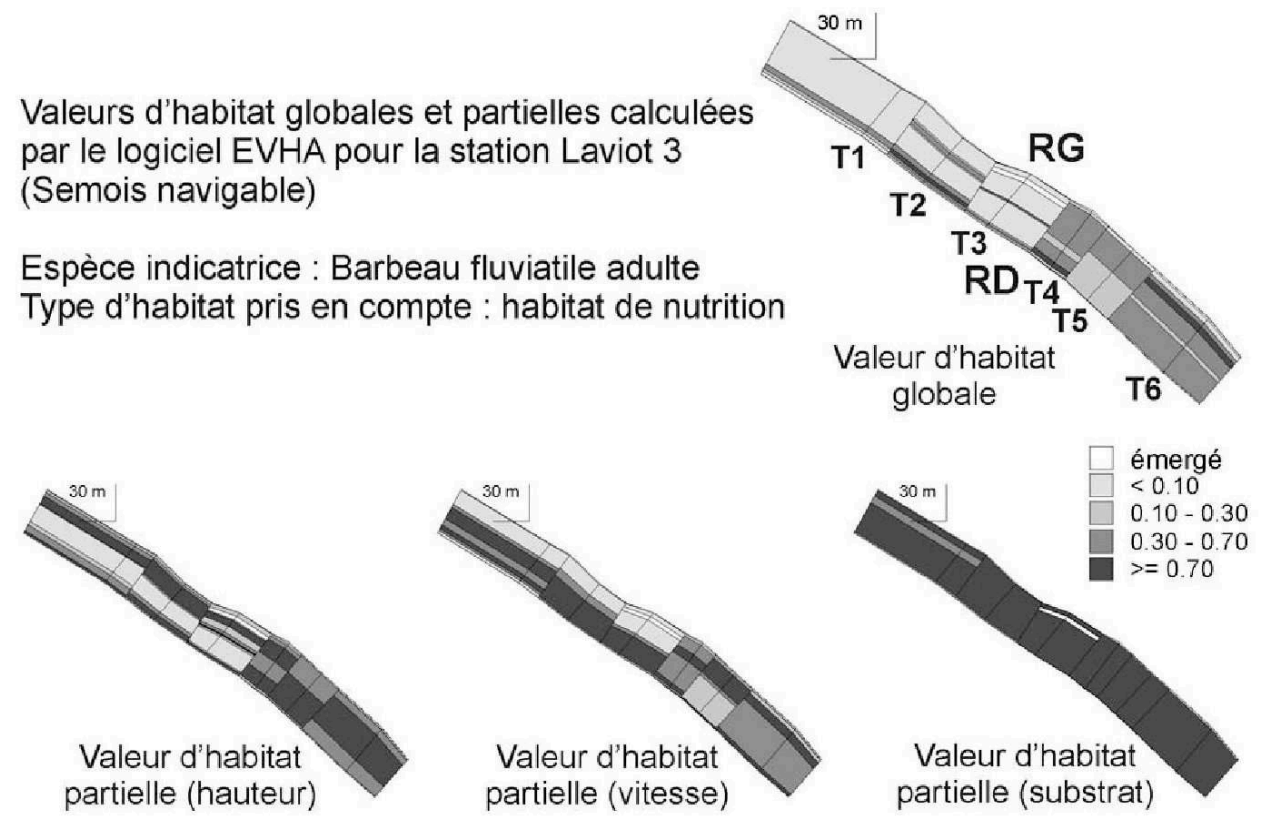

Les valeurs d'habitat sont représentées par un indice compris entre 0 et 1

Le curage effectué à Laviot en 1997 sur une demi-largeur de la rivière en rive droite a notamment perturbé un faciès du cours d'eau qui constitue l'habitat de nutrition du barbeau adulte (faciès de plat). Les graphiques d'estimation de la SPU et des valeurs d'habitat pour cette espèce obtenus par EVHA sont présentés aux figures 8 et 9. Ils mettent clairement en évidence une SPU moindre et une valeur d'habitat globale très faible (< à 0.10) pour la partie amont (transects 1 à 3) qui a subi le curage par rapport à la partie aval non touchée (transects 4 à 6). Ce constat s'explique en regardant les valeurs d'habitat partielles obtenues pour chacune des 3 variables physiques. L'augmentation de la profondeur ainsi que la modification des vitesses latérales 
générées par la chenalisation se caractérisent par des valeurs d'habitat très faibles pour l'habitat de nutrition du barbeau adulte au niveau de cette zone curée. Cette perte d'un potentiel d'habitat pour le barbeau adulte considéré dans sa fonction de nutrition a également été confirmée par comparaison avec les résultats obtenus par EVHA sur un linéaire de la Semois qui n'a pas fait l'objet du curage de 1997 et présentant le même type de faciès, à savoir le plat «lentique » et qui n'a pas fait l'objet du curage de 1997. Le faciès "lentique" se définit par des hauteurs d'eau faibles et uniformes sur l'ensemble de l'unité, des vitesses moyennes et également très uniformes, une pente ligne d'eau souvent parallèle à celle du fond et un écoulement uniforme avec peu ou pas de vaguelette à la surface de l'eau (Malavoi, 1989).

Cette méthodologie apparaît très pertinente. Son application avant et après de futurs travaux permettrait d'apprécier de manière précise la perte de capacité d'accueil du cours d'eau pour l'ichtyofaune. Cependant, elle se limite pour l'instant uniquement aux variables morphodynamiques, c'est à dire celles qui dépendent de la morphologie de la rivière et de son débit. Cela signifie en particulier que les variables physico-chimiques, et parmi elles la température ou l'oxygène, sont exclues du modèle, ainsi que les polluants. Il est pourtant bien évident que ces variables ont une influence importante sur les conditions de vie du poisson (son biotope) et qu'elles sont également fortement dépendantes du débit. Mais leur évolution se fait à l'échelle de l'ensemble du bassin amont de la rivière et il est impossible de les simuler en restant à l'échelle de la station comme le fait la méthode des microhabitats. Ces variables font donc partie du contexte écologique global à ne pas oublier dans l'interprétation des résultats.

D'autres variables de l'habitat pourraient être intégrés dans cette modélisation, et notamment la présence des abris (sous-berges, embâcles...), dont l'importance écologique a été soulignée par de nombreuses études sur presque tous les types de cours d'eau (Binns et Eisermann, 1979 ; Bovee, 1982 ; Heggenes et al., 1990 ; Pouilly, 1994). Dans certains cas également, la végétation immergée peut devenir une composante importante de l'habitat (cas des renoncules flottantes dans la Semois).

Une extrapolation des résultats à une échelle plus large, et en particulier à toute la portion de rivière étudiée, est possible ; elle doit se baser notamment sur l'approche de sectorisation en faciès morpho-écologiques en reproduisant de manière significative la méthode sur les types de faciès rencontrés dans la rivière. Signalons à ce sujet, qu'à l'échelle d'analyse du tronçon, d'autres outils pourraient être testés (STATHAB et ESTIMHAB développés par le CEMAGREF de Lyon (Ginot et al., 1998)) afin de caractériser l'habitat piscicole. Pour ces deux outils, la modélisation de l'hydraulique est statistique et non plus au moyen d'un modèle 1D qui supposait un calage topographique de la ligne d'eau. Le protocole de collecte des données ne demande pas de repérages topographiques des points de mesures mais impose deux campagnes d'acquisition des données à des débits différents. Le couplage est fait pour le cortège d'espèces connues sur le site, avec des courbes de préférence multivariées; l'interprétation analyse les tendances d'évolution du peuplement.

Une approche plus fine de certains paramètres (sédiments fins...) ou bioindicateurs (macro-invertébrés...) directement en lien avec la problématique d'estimation des impacts écologiques générés par les travaux d'aménagement du lit mineur et des berges en Basse-Semois pourrait également être envisagée dans une poursuite de cette étude. 


\section{Conclusion}

\section{BIBLIOGRAPHIE}

BALON E.K. (1975), « Reproductive guilds of fishes: a proposal and definition », Journal of the Fisheries Research Board of Canada, 32, pp. 821-864.

BARAS E. (1992), «Etude des stratégies d'occupation du temps et de l'espace chez le barbeau fluviatile, Barbus barbus (L.) ", Cahiers d'Ethologie Appliquée, 12, 2-3, pp. 125-442.

BERTRAND G. (1994), Le calcul des axes hydrauliques dans des rivières à plaine inondable, mémoire de stage, Laboratoire de Recherches hydrauliques de Châtelet.

BINNS N.A., EISERMANN F.M. (1979), « Quantification of fluvial trout habitat in Wyoming », Transactions of American Fisheries Society, 108, 3, pp. 215-228.

BOVEE K.D. (1982), A guide to stream habitat analysis using the Instream Flow Incremental Methodology, U.S.D.I. Fish and Wildlife Service, Office of Biological Services, Fort Collins, Colorado, 248 p.

CUNGE J.A., HOLLY F.M.JR \& VERVEY A. (1980), Practical aspect of computational river hydraulics, Pitman Publishers, London, $420 \mathrm{p}$.

GINOT V. \& SOUCHON Y. (1998), Logiciel EHVA, Evaluation de l'habitat physique des poissons en rivière. vol. 1. Guide méthodologique. vol. 2. Guide de l'utilisateur, Cemagref Lyon BEA/LHQ, France, et Ministère de l'Environnement, Dir. de l'Eau, Paris, France, 87 p. 
HEGGENES J., BRABANT A., SALVEIT S.J. (1990), « A comparaison of three different methods for studies of stream habitat use by brown trout and Atlantic salmon », Transactions of American Fisheries Society, 109, pp. 101-111.

HUET M. (1949). « Aperçu des relations entre la pente et les populations piscicoles des eaux courantes ", Revue Suisse d'Hydrologie, 11, 3-4, pp. 332-351.

HUET M. \& TIMMERMANS J.A. (1976), Influence sur les populations de poissons des aménagements hydrauliques de petits cours d'eau assez rapides, Trav. Série D, $\mathrm{N}^{\circ} 46$, Ministère de l'Agriculture, Station Rech. des Eaux et Forêts de Groenendaal-Hoeilaart, Belgique, 27 p.

MALAVOI J.R. (1989), « Typologie des faciès d'écoulement ou unités morphodynamiques des cours d'eau à haute énergie ", Bulletin Français de la Pêche et de la Pisciculture, 15, pp. 189-210.

PETIT F., PAUQUET A. \& PISSART A. (1996), « Fréquence et importance du charriage dans des rivières à charge de fond caillouteuse ", Géomorphologie : Relief, processus, environnement, 2, pp. 3-12.

PHILLIPART J.C., BARAS E., NINDABA J. (1994), Etude des aspects hydrobiologiques et écologiques des dragages et des aménagements hydrauliques dans une rivière de type Ourthe, Rapport d'étude au Ministère de l'Equipement et des Transports (MET) de la Région Wallonne (DGRNE), Laboratoire Démographie des Poissons et d'Aquaculture, Université de Liège, 143 p. + annexes.

POUILLY M. (1994), Relations entre l'habitat physique et les poissons des zones à cyprinidés rhéophiles dans trois cours d'eau du bassin rhodanien : vers une simulation de la capacité d'accueil pour les peuplements, Thèse de doctorat, Université Claude Bernard - Lyon I, 147, 94, 256 p.

STALNAKER C.B. (1979), « The use of habitat structure preferenda for establishing flow regimes necessary for maintenance of fish habitat », in Ward \& Stanford (eds.), The Ecology of Regulated Streams, Plenum Press, New York, pp. 326-337.

\section{RÉSUMÉS}

Cette étude tente d'évaluer les impacts des dragages réalisés dans le bassin de la Semois (Ardenne). Cette une étude interdisciplinaire se penche sur les aspects hydrauliques, environnementaux, géomorphologiques et sédimentologiques. L'étude géomorphologique et sédimentologique s'axe sur l'évolution de la dynamique fluviale via l'étude de carte et de photos anciennes. Une analyse du transport de la charge de fond de la rivière a aussi été menée afin de quantifier le taux de remblayage des différents dragages. L'aspect hydraulique se résume à l'application d'un modèle hydraulique (Bertrand, 1994) dans le but de cerner les effets des dragages sur le niveau d'eau. L'aspect environnemental consiste à évaluer les pertes d'habitabilité du fond du lit suite aux différents dragages grâce à l'usage du logiciel EVHA. Au final, les différents dragages ont eu peu d'effet en ce qui concerne la protection contre les inondations. Les temps de retour à la situation initiale ne dépassent pas 10 ans. Les pertes d'habitabilité pour la faune aquatique sont significativement importantes dans les sites dragués.

This study aims to evaluate impacts of riverbed dredgings in the Semois catchment (Ardenne). This is an interdisciplinary study focused on the hydraulic, environmental, geomorphological and sedimentological aspects. The geomorphological and sedimentological study compare old maps and old photograph to highlight the general evolution of the river. Also, the bedload discharge has been calculated. A hydraulic model, (Bertrand, 1994) has been applied to evaluate the effect of dredging on the water level. The software EVHA has been used to evaluate the losses of fish's habitat in the dredged sites. The dredging have low effect terms of flood protection and 
the time needed to recover the pre-works situation, and such works has reduced the environmental index in the dredged sites.

\section{INDEX}

Mots-clés : dragage, sédimentologie, charge de fond, hydraulique, ichtyofaune, barbeau fluviatile

Keywords : dredging, sedimentology, bedload, hydraulics, ichtyofauna, barbel

\section{AUTEURS}

\section{F. GOB}

Université de Liège, Laboratoire d'Hydrographie et de Géomorphologie fluviatile, fgob@ulg.ac.be

\section{G. HOUBRECHTS}

Université de Liège, Laboratoire d'Hydrographie et de Géomorphologie fluviatile, G.Houbrechts@ulg.ac.be

\section{J. MOLS}

Université de Liège, Laboratoire d'Hydrographie et de Géomorphologie fluviatile, jmols@ulg.ac.be

\section{F. PETIT}

Université de Liège, Laboratoire d'Hydrographie et de Géomorphologie fluviatile, François.Petit@ulg.ac.be

\section{F. GUYON}

Fondation Universitaire Luxembourgeoise, Guyon@ful.ac.be

\section{F. ROSILLON}

Fondation Universitaire Luxembourgeoise, rosillon@ful.ac.be

\section{COGELS}

Fondation Universitaire Luxembourgeoise, cogels@ful.ac.be

\section{P. VANDER BORGHT}

Fondation Universitaire Luxembourgeoise, vdborght@ful.ac.be

\section{J.P. ANCION}

Ministère de l'Equipement et des Transports, Laboratoire de Recherche Hydraulique (Châtelet), jpansion@met.wallonie.be

\section{E. NTIBARUFATA}

Ministère de l'Equipement et des Transports, Laboratoire de Recherche Hydraulique (Châtelet), entibaru@met.wallonie.be 


\section{J.M. HIVER}

Ministère de l'Equipement et des Transports, Laboratoire de Recherche Hydraulique (Châtelet), jmhiver@met.wallonie.be

\section{J.C. PHILIPPART}

Université de Liège, Laboratoire de Démographie des Poissons et d'Hydroécologie, jcphilippart@ulg.ac.be 